\title{
Estrategia de integración de los agentes educativos que intervienen en la formación integral de los estudiantes becarios de la Universidad de Holguín Oscar Lucero Moya
}

\section{Strategy for the integration of teaching agents involved in boarding students' integrated education at Oscar Lucero Moya University}

\author{
Aida Luisa Narbona Gutiérrez \\ CASED \\ Universidad de Holguín Oscar Lucero Moya, Cuba \\ anarbona@ict.uho.edu.cu \\ Amable Faedo Borges \\ Asesor de Ciencia e Innovación Tecnológica del Grupo de Trabajo Educativo \\ Universidad de Holguín Oscar Lucero Moya, Cuba \\ afaedo@ict.uho.edu.cu
}

Recibido: 30-VIII-2011 • Aceptado: 06-X-2011 • Corregido: 15-VI-2012

\begin{abstract}
Resumen: Se ofrece una estrategia para la integración de los agentes educativos que influyen en la formación y desarrollo de los estudiantes. Se demuestra la importancia de perfeccionar el trabajo educativo en la residencia estudiantil a partir del enfoque estratégico integrador. Este aporte posibilita aunar a los agentes educativos, al proponer acciones que facilitan su aplicación y puesta en práctica, con la finalidad de perfeccionar el trabajo educativo e influir en el estudio teórico y metodológico de este enfoque en la Residencia Estudiantil de la Universidad de Holguín Oscar Lucero Moya y las transformaciones que hoy enfrenta la enseñanza superior.
\end{abstract}

Palabras clave: Estrategia de integración, formación integral, agentes educativos.

Abstract: The paper focuses on a strategy for the integration of teaching agents that influence boarding

\section{Introducción}

La formación integral del futuro profesional no constituye una preocupación privativa del contexto cubano, lo cual ha sido expuesto en diversos documentos y publicaciones compendiadas y así lo aseguran un conjunto de investigadores: [The Economist en 1990, publicó una investigación acerca del tema de Visión, Misión y Valores, en la que evalúa las culturas organizacionales más fuertes y con mayor coherencia en la práctica de valores, esta publicación integra el aporte de diferentes investigaciones como: Charles Hill y Gareth Jones (1990) y Jack Haas (1990) ${ }^{1}$ ], Ken Blanchard y Michael OConnor (1997), 
students' education and development. The importance of improving the work of teaching agents with boarding students, through an integrated strategic approach is shown. This contribution favors the integration of teaching agents, suggesting actions that facilitate its application for the improvement of teaching. It also influences theoretical and methodological studies in the field of students' halls of residence according to current higher education transformations.

Keywords: Integration strategy, Holistic education, teaching agents.
Schukina G.I. (1982), Boldiriev N.I. (1982), Corso Fabelo (2000), Carlos Díaz (2002), Gilberto García (2002), Esther Baster (2002), Pedro Hourruitinier (2006), entre otros. Este hecho emerge en países del primer mundo y también en los menos desarrollados económicamente.

Hoy en día la Organización de Estados Iberoamericanos (OEI) invierte significativamente en la dirección estratégica de valores, teniendo en cuenta la repercusión global de este hecho en la sociedad. Por ello, es ineludible indagar en hallazgos que necesariamente no tienen que formar soluciones tecnológicas, pero sí se presentan bien estructurados y pueden convertirse en la solución de hechos que precisan ser investigados por la ciencia.

Por ello se torna indispensable que el proceso de formación sea asumido por todos, interviniendo con una visión integradora estudiantes, trabajadores y las diferentes formas de organización de trabajo educativo existentes, además de las estructuras organizativas: universidad, facultades y departamentos.

En este texto se trata el caso de un contexto educativo por excelencia, la residencia estudiantil, la cual posee una estrecha vinculación con un proceso que comienza en la docencia y se expresa en la vida social. Este es un espacio donde se refuerzan hábitos, habilidades, cualidades y valores como el amor a la patria, colectivismo, responsabilidad y amistad.

Este escenario educativo constituye un facilitador para el estudio de la subjetividad del individuo, propiciador de éxito del control confiable y la evaluación objetiva de los resultados desde la corrección de la planificación y organización de las actividades dirigidas a un objetivo de carácter instructivo-educativo, incluyendo también la conformación de su tiempo libre, favoreciendo así el desarrollo integral de los educandos.

En la educación cubana se observan cambios significativos en sus aspiraciones de extensión y se descubren contradicciones entre la teoría y la práctica, la cual enuncia 
que para educar al ser humano en el logro de una incorporación personal y socialmente activa es necesario integrar todos los escenarios y agentes educativos. La práctica desde esta perspectiva demuestra impasibilidad al reducir estos escenarios a una rutina tradicional arraigada que imposibilita cambios en su funcionamiento interno.

Considerando las exigencias que se plantean en la enseñanza superior y las transformaciones que en ella se llevan a cabo, es objetivo de este trabajo: socializar una estrategia de integración de los agentes educativos que intervienen en la formación integral de los estudiantes becarios de la Universidad de Holguín Oscar Lucero Moya. Estrategia que puede ser generalizada a nivel de otras instituciones, pues propone un conjunto de acciones que facilitan su aplicación práctica a partir del enfoque estratégico integrador del mismo.

\section{Desarrollo}

\section{Breves fundamentos teóricos del trabajo educativo}

A la enseñanza superior le corresponde enfrentar los desafíos que hoy le impone la sociedad y en sus aspiraciones de extensión se hace imprescindible aunar esfuerzos para alcanzarlos. Los cambios y transformaciones que requiere la educación superior no son solo estructurales, sino conceptuales, de procedimientos y sin dudas de mucha convicción de la necesidad del cambio y de su renovado compromiso social. Estas ideas expresadas por Juan Vela (2008) convocan a cumplir el rol que corresponde a cada persona en estos momentos de profundos cambios y a concienciar el encargo social que posee la enseñanza superior. Todo esto requiere cambios trascendentales en la forma de dirigir el proceso educativo en esta institución educacional.

La formación de hombres y mujeres, como la definen los estudiosos del tema, Makarenco A.S., Schukina G.I.
(1982), Boldiriev N.I. (1882), Gilberto García (2002), Esther Baster (2002), Pedro Hourruitinier (2002), entre otros, coinciden que es un proceso continuo y complejo que requiere de precisión en los objetivos de carácter educativo a lograr y determinación de las cualidades de la personalidad que se han de formar y desarrollar, teniendo en cuenta la edad y grupo en que se trabaja. Los autores además plantean la importancia de la escuela, como institución social, basados en el criterio de que es en ella donde se realiza el proceso educativo, caracterizándolo por un conjunto dinámico y complejo de actividades sistemáticas, mediante el cual se interrelaciona la educación de los educandos, en que dichas actividades están encaminadas tanto a la educación del grupo, como a la de cada uno de sus miembros.

El éxito de la actividad educativa depende en gran medida del control adecuado y de la evaluación objetiva de sus resultados, partiendo de la corrección de la planificación y organización de las tareas educativas.

Juzgar los resultados por la cantidad de actividades que se realizan o por la cantidad de actividades en las cuales participan los estudiantes constituye una equivocación, esto no implica que no sea necesario llevar el control de estas actividades, pero lo anterior impide evaluar de forma apropiada y sistemática los resultados de la actividad educativa y establecer cómo ha influido esta en el desarrollo de la personalidad. Por ello es mucho más importante determinar cómo han influido las mismas sobre el desarrollo de los alumnos, los cambios que han ocurrido en su conciencia, su conducta y cuáles son las cualidades de la personalidad que se han formado o modificado satisfactoriamente, residiendo aquí la complejidad de este proceso.

Cuando se evalúa el nivel de desarrollo de una cualidad en los alumnos no basta con aplicar parámetros con enfoques cuantitativos, se necesita ante todo índices cualitativos que permitan caracterizar el 
nivel de educación de los educandos. Estos niveles se manifiestan en los hábitos estables de conductas, en los actos y acciones de los alumnos y en su seguimiento regular para no obtener como resultado simulaciones conductuales.

Para poder comprender a los alumnos hay que saber cuáles son los motivos de su conducta, que a veces no se pueden observar directamente. Estos también los impulsan a comportarse de determinada forma, pues la conducta aparente del individuo no expresa su estado interior $\mathrm{y}$ en ocasiones contradice sus verdaderas emociones. No debe juzgarse sobre el nivel de educación de los educandos, sino por los resultados de su actividad en relación con las otras personas. El nivel de educación debe comprobarse cada día, cada hora, considerando cualquier acto del alumno, por ejemplo su conducta y sus actitudes, permitiendo caracterizar la educación de este, lo que se traduce en ofrecer una evaluación confiable del comportamiento de estos en diferentes condiciones de la vida.

Comprobar y evaluar los resultados de la educación es más complejo que evaluar los de la enseñanza, pues para determinar y evaluar el conocimiento de los alumnos basta con sondearlos o proponerles una situación que implique su resolución, mediante el control. Los resultados de la educación son más complejos, pues no pueden determinarse por la vía de la instrucción, para esto se requiere largo tiempo y se recurre a la utilización de otros métodos y procedimientos.

En la educación superior, Pedro Hourruitinier (2006), declara que la unidad entre el proceso de instrucción y el de educación es el principio en el que se sustenta la labor educativa, es decir, se instruye y se educa en todo momento, mediante la participación activa y consciente del educando en las diversas actividades. Esto con la exigencia de que para lograr un trabajo educativo con resultados satisfactorios es fundamental tener en cuenta lo siguiente: los objetivos educativos propuestos, las necesidades individuales y grupales, las de realizar una actividad y no otra, todo en función de sus características y edades, lo que determina su participación en la elección de las actividades, las condiciones concretas de la escuela y la comunidad.

Es de gran importancia para el enfoque integrador y la concreción de los propósitos permanentes de la Revolución Socialista Cubana que el profesional sea de elogiados conocimientos, no solo de su especialidad, sino que también sea conocedor del estado del arte, de los principales hallazgos científicos y tecnológicos que acontecen en el mundo, capaz de disertar sobre temas de política, artes plásticas, música, sexualidad, entre otras manifestaciones existentes que generan integralidad en valores culturales. Se busca lo anterior ya que permite aunar al colectivo y obtener un plan único de trabajo, una forma única de abordar al joven para que pueda haber proceso educativo, por lo que deben encontrarse insertadas todas las estructuras y formas organizativas de la universidad, implicando las direcciones de áreas de resultados claves hasta la brigada estudiantil.

La labor educativa en el contexto de la Residencia Estudiantil para la formación de los estudiantes no es solamente responsabilidad de los educadores que laboran ahí, sino de todos los profesores y trabajadores de la institución, que desde sus diferentes responsabilidades deben asumir constantemente el objetivo fundamental de la educación superior. Esto desde cualquier función que realicen, expresando dicha responsabilidad en manifestaciones comportamentales de la ejemplaridad, la cultura y la intervención oportuna ante las ilegalidades, entre otros aspectos.

Cuando no se tiene conciencia de la importancia de la labor educativa en esta etapa, en la cual su culminación está vinculada a perfilar la vida adulta del joven $\mathrm{y}$ fundamentalmente la actividad laboral para la que se ha preparado durante todo su tránsito por las diversas enseñanzas, esto trae consigo que no se comprometan 
con una serie de deberes y derechos que deben enfrentar, asignados por la sociedad para que finalmente tengan que comportarse con mayor autonomía, madurez y responsabilidad.

Este edificio es más que una construcción: constituye el espacio educativo más importante en el seno de una institución que brinda servicios a una población heterogénea de disímiles municipios, provincias y nacionalidades para contribuir a la formación de hábitos y habilidades que le permitan al futuro profesional la asimilación de normas de conducta, sentimientos, cualidades, actitudes de conceptos morales, de valores, principios, convicciones, haciéndolo partícipe consecuentemente de la práctica activa y creadora en el contexto del proceso histórico social en el que se desarrolla.

\section{Metodología y resultados}

Para poder conocer la realidad educativa de la Residencia Estudiantil, con vistas a su estudio, se utilizaron un conjunto de métodos de investigación, encontrándose dentro de los métodos teóricos los siguientes:

Histórico-lógico: con el objetivo de valorar los antecedentes históricos y las transformaciones que hoy enfrenta la enseñanza superior, con énfasis en el trabajo de la residencia estudiantil. Se utilizaron en el procesamiento de la información tanto teórica como empírica el análisis y la síntesis y la inducción-deducción, lo que permitió la caracterización del objeto de investigación, la determinación de los fundamentos teóricos y metodológicos, la elaboración de las conclusiones y el método de modelación, dirigido a la concepción y estructuración de la estrategia integradora y a que esta contuviera los elementos necesarios para darle carácter participativo, flexible, sistémico e integrador.

Dentro de los métodos empíricos se trabajó con la observación, prestando atención a diferentes procesos en los que intervienen estudiantes y agentes educativos para la detección de fortalezas y debilidades, pudiendo apreciar los siguientes resultados:

El personal docente que interviene de forma directa en la formación de los futuros profesionales goza de gran prestigio desde el punto de vista de sus conocimientos en el plano tecnológico, alcanzando maestría en su labor por su constante preparación y dedicación, pero no posee las herramientas necesarias para la realización del trabajo educativo por lo que debe profundizarse en su formación pedagógica.

De los veinte docentes escogidos como muestra, solo tres mostraron disposición para realizar la actividad para un $15 \%$, los 17 restantes realizaron la actividad, pero sin mostrar disposición, indicando malestar hacia la labor para un $85 \%$.

Esta observación reveló que la información que utilizaron para la realización de la actividad fue obtenida mediante las administradoras del edificio y grupo de trabajo educativo.

La utilización de esta técnica demostró que siempre es el mismo profesor quien realiza esta actividad a nivel de las distintas carreras, afectando la estabilidad de los resultados de la realización de esta.

En las visitas realizadas a los cuartos del estudiantado de la totalidad de docentes observados, solo ocho lograron el objetivo que se propusieron en las actividades de apoyo al trabajo educativo de la residencia para un $40 \%$, los restantes se limitaron a las charlas, sin obtener los resultados deseados, representando el $90 \%$.

De los 250 estudiantes observados solo el 14\% manifestaron complacencia en la realización de las actividades reglamentadas, el 86\% de los restantes adoptaron actitudes no acordes con la necesidad de la realización de estas importantes tareas.

Los cuartos no resultan el lugar más higiénico y acogedor para su descanso, manifestándose conductas inapropiadas como tirar basura a las áreas verdes; además, no se observa en los cuartos una 
adecuada decoración que haga su estancia agradable, constatándose que no existe una consideración por los medios y materiales puestos a su disposición, acrecentándose las roturas, manifestaciones de hurtos del equipamiento brindado para su bienestar y observándose asimismo la presencia de personal ajeno al centro.

El vocabulario utilizado por los becarios no se corresponde con su nivel de instrucción con manifestaciones que tienden a la vulgaridad, utilización de tono de voz inadecuado, no identificándose aún con los objetivos que persigue la Residencia Estudiantil, evidenciándose en ellos todavía una pobre cultura general, elementos que resultan prioritarios en los objetivos de trabajo educativo.

Por su parte, la entrevista se explotó para conocer motivos, necesidades e intereses de los estudiantes, además para conocer cómo intervienen los diferentes agentes educativos en la realización de la labor educativa. Expresándose de esta manera los siguientes resultados:

En la técnica aplicada a los docentes para constatar sus habilidades en el diagnóstico el $100 \%$ coincidió en que lo aplicaban basándose solo en la identificación de dificultades a nivel cuantitativo, sin poder explicar las causas que originaban determinada conducta del estudiante.

$\mathrm{Al}$ estudiar en qué medida consideran al claustro de profesores preparados para asumir la labor educativa desde la residencia, los veinte docentes encuestados coincidieron en sus criterios: no poseían conocimientos sólidos para realizar el diagnóstico, existieron dificultades que presentaban sus estudiantes y no podían darles solución, además de que los limitaba la gran carga docente que tenían.

Se indagó acerca de las acciones que llevaban a cabo con respecto a la Residencia Estudiantil y la totalidad coincidió en la realización de visitas a los cuartos para conversar con los alumnos y realizar charlas con el objetivo de que realizaran la limpieza y labores de cuartelería.
Cuando se les preguntó acerca de la dimensión del proceso evaluativo de índole educativo para orientar y controlar la labor educativa en la Residencia Estudiantil, que coadyuvara a la formación del estudiante, expresaron que había la participación del departamento de Extensión Universitaria en actividades como conferencias de VIH, talleres sobre drogas y la asistencia a los Consejos de Residencia.

Con relación al control y evaluación que ejercen los docentes en estas acciones, los profesionales entrevistados aportaron que ya ello constituía una exigencia en la facultad y que se elaboraban acciones con este fin.

Por otra parte, la entrevista aplicada a los estudiantes para evaluar las relaciones de estos en los cuartos manifestaron que poseen buenas relaciones con sus compañeros, que no se metía en nada, que cuando necesitaban de ellos podían contar y que influían en su grupo ayudando a los otros y que los justificaban si no limpiaban o no iban al aula, que no tenían ningún interés de que estos cambiaran en nada y que si tuvieran que estar becados de nuevo les gustaría estar con el mismo colectivo, teniendo confianza en ellos. Entre los alumnos, 65 expresaron la inexistencia de usurpadores a pesar de que ha habido hurtos, pero que no se habían visto afectados, el resto de los estudiantes si se han visto afectados por ese delito producido en el edificio para un $26 \%$.

También, 200 estudiantes encuestados opinaron que la guardia estudiantil en el cuarto no resulta necesaria, demostrando la no relación del hecho delictivo que más afecta, el hurto, y la participación activa de ellos para su decrecimiento (80\%), y solo 50 reclaman la necesidad de tomar decisiones para solucionar esta dificultad (20\%).

De los entrevistados, 250 invierten en estudiar, expresando que las clases son muy difíciles y los profesores muy exigentes.

Las relaciones que mantienen con sus profesores, según lo expuesto, resulta satisfactoria, alegan que son buenos, que se encuentran muy preparados y que se 
preocupan por ellos y los exigen en las asignaturas que imparten.

Coinciden en que los profesores vienen a visitarlos a los cuartos y que son constantes en el cumplimiento de esa actividad 138, para un 55,2\%, 30 expusieron que casi siempre, $12 \%$ y 82 que no lo hacen para el $32,8 \%$.

La encuesta se aplicó a los estudiantes para poder recoger información sobre las características de las relaciones interpersonales e identificar sus motivos, necesidades e intereses.

$\mathrm{Al}$ analizarlas, se aprecian buenas relaciones y expresión de unidad, en que el vínculo fundamental es la profesión. La influencia ejercida por los dirigentes del grupo resulta favorable, apreciándose por las constantes muestras de cariño y admiración por la inteligencia y eficiencia de sus líderes.

Además, se demostró en la totalidad de las respuestas enunciadas de la encuesta aplicada la existencia de estudiantes no aceptados en el cuarto por predominar en estos rasgos perfeccionistas de autosuficiencia y de homosexualidad, aclarando que a pesar de esto realizan el mayor esfuerzo por aceptarlos.

Los estudiantes más aceptados en el cuarto son aquellos que ayudan a los demás y no se dedican a realizar comentarios que puedan afectar la integridad del grupo, su criterio siempre es de subordinación asociándose a todo lo que dice y hace el grupo, criterio final muy nocivo pues esto implica aceptación de conductas censurables.

En la totalidad de opiniones recogidas los estudiantes muestran sentimientos de alegría y placer, se sienten aceptados y necesarios por los miembros del cuarto, expresando que se les pide consejo a la hora de tomar una decisión importante e identificándose con los problemas de cada uno de ellos.

El 100\% de los becarios contribuye en su accionar diario a mantener la unidad de su cuarto: respetándose unos a otros, ayudándose, aceptándose con sus virtudes y defectos, indicándose cada uno en qué está afectando el grupo para evitar dividirse.

Confrontando los criterios expuestos por los pedagogos universitarios y los estudiantes becarios, se logró identificar algunas de las causas más importantes que sustentan las insuficiencias en la educación de los estudiantes expresadas fundamentalmente: en el distanciamiento existente entre los docentes y el Grupo de Trabajo Educativo, no conciliando estos acciones que contribuyan a la formación integral de los educandos, manifestándose un tradicionalismo en la planificación, organización y evaluación de este proceso.

El criterio de profesionales, para buscar criterios de consenso sobre la pertinencia de la estrategia propuesta y el método matemático, contiene el procesamiento de los datos de las encuestas y entrevistas.

La utilización armónica de cada uno de estos métodos permitió evaluar el nivel de desarrollo de los futuros profesionales, la preparación y eficacia del personal profesional encargado de perfeccionar su desarrollo y el estado actual del proceso educativo en la Residencia Estudiantil. Los resultados obtenidos fueron comparados con acciones transitadas y vigentes, bibliografías, estrategias, aportando presupuestos teóricos para la fundamentación y detección de fortalezas, debilidades y amenazas.

\section{Modelación de la estrategia de integración}

La estrategia que se propone tiene su principal fundamento en las concepciones teóricas y metodológicas del trabajo educativo, además de los postulados de la política educacional cubana acerca de las transformaciones de la escuela y en particular de la enseñanza superior con enfoque integrador. El modelo metodológico para la elaboración de esta estrategia es declarado por Alejandro Augier (2000).

En esta primera etapa de elaboración de la estrategia, predominan decisiones 
de carácter estratégico, de cumplimiento a largo plazo, y sus resultados principales son la definición de la misión, la visión y los objetivos estratégicos.

La segunda etapa, comprende la implementación de la estrategia y es en esta etapa donde se concretan los resultados para un período más breve que la primera fase, y posibilita el cumplimiento parcial de los objetivos estratégicos planteados.

Estos resultados tienen su expresión en el establecimiento del plan anual para un curso escolar, definiendo los objetivos tácticos y operativos derivados de los estratégicos y el sistema de acciones con el que se les dará cumplimiento.

Estrategia de integración de los agentes educativos que intervienen en la formación integral de los estudiantes becarios de la Universidad de Holguín Oscar Lucero Moya.

Misión: La residencia estudiantil de la Universidad de Holguín Oscar Lucero Moya posee la misión de ofrecer servicios de primera necesidad y de apoyo a la labor educativa en función de la formación integral, tanto a estudiantes becarios nacionales como extranjeros.

Visión: Afianzamiento de la labor en la Residencia Estudiantil como espacio esencialmente educativo, donde se fortalezcan en los estudiantes cualidades, valores morales y hábitos como convivencia, solidaridad, disciplina, cuidado a la propiedad social, entre otras.

Objetivo de la residencia estudiantil: Contribuir a la elevación del nivel educativo de los estudiantes becarios mediante la integración de los agentes educativos que intervienen en su formación.

Estado deseado: Establecer interrelación con los agentes educativos que intervienen en la formación integral de los estudiantes, con el fin de contribuir al fortalecimiento de cualidades, valores morales, hábitos, entre otros, y que para la solución de los problemas existentes prevalezca el protagonismo estudiantil.
Plan de acciones:

1. Instrumentación y sistematización del diagnóstico psicopedagógico individual y grupal: Esto enfatizando en las siguientes dimensiones: desarrollo físico y estado de salud, aprendizaje, esfera afectivo-volitiva, desarrollo ideológico-moral, desarrollo laboralprofesional, desarrollo psicosexual y proyección hacia sí mismo.

2. Preparación de cursos dirigidos al accionar con el diagnóstico: Esto con la finalidad de confeccionar los indicadores y orientar a los implicados en esta tarea. Entre los contenidos a tratar acorde con las fortalezas e insuficiencias que pueden presentar los participantes, se encuentran: definición de diagnóstico psicopedagógico integral; principios del diagnóstico; métodos para la obtención de informaciones sobre el estado del problema y dimensiones e indicadores de diagnóstico.

3. Creación de documentos metodológicos complementarios: dirigidos a la educación del estudiante en temas como medio ambiente, salud, valores éticos y estéticos para una convivencia armónica, ética y estilo de relación entre los profesionales, apreciación del arte, política, entre otros.

4. Creación de programas de postgrado sobre fundamentos psicológicos y pedagógicos de la dirección de la labor educativa con los estudiantes becarios: Esto para docentes de las carreras y profesores educativos de la Residencia.

5. Formación y sistematización en los estudiantes y profesores de una educación jurídica: Particularmente en el cumplimiento de forma consciente del reglamento y compromiso del becario en la Residencia Estudiantil para poder entender lo estipulado reglamentariamente y en qué consiste su procedimiento, con el objetivo 
de llegar a su aplicación y a la práctica consciente del estudiante. Se puede proceder mediante los temas de la preparación política establecidos, sesiones especiales de debate por las brigadas estudiantiles y sesiones sindicales, entre otras variantes.

6. Capacitación de las estructuras políticas y estudiantiles en la Residencia Estudiantil: Con el fin de fortalecer las estructuras políticas y dirigentes de base de la FEU, UJC, en la residencia estudiantil, en contenidos de ética, cortesía, educación formal, educación sexual, entre otros. 7. Visitar con frecuencia a los estudiantes en su medio más informal: Para conocer cómo viven, cuáles son sus inquietudes, entre otras cuestiones de interés, y de esta forma acercarse más a su realidad. Estas visitas se deben programar en los planes de trabajo de las organizaciones para evitar improvisaciones y lograr la participación conjunta en las mismas, aún cuando algunas se puedan realizar de forma sorpresiva. 8. Realización de trabajo preventivo: Con el conocimiento obtenido sobre el comportamiento del estudiante, se emplearían acciones dirigidas a la formación de la conciencia moral, teniendo en cuenta la trascendencia social de la indisciplina; para estimular, alertar, prevenir, convencer y comprometer dicho estudiante, sin llegar a la aplicación del reglamento. Esta tarea se desarrollará mediante consultorías y sesiones de trabajo individual y grupal por el grupo de trabajo educativo y profesores guías.

9. Creación de grupos científicos: Con el objetivo de solucionar a corto, mediano y largo plazo las dificultades existentes en el banco de problemas de la Residencia Estudiantil proporcionándoles la oportunidad de desarrollar actividades y proponer soluciones que tengan para los educandos sentido personal y social. El contenido y la forma de organización de esta actividad deben subordinarse a las tareas docentes educativas de la institución. El grupo de trabajo educativo de la Residencia Estudiantil debe ser un colaborador activo de esta actividad, identificando las fortalezas, debilidades y amenazas existentes en la residencia, adecuándolas en las líneas investigativas y verificando el cumplimiento de la actividad.

10. Participación del grupo de trabajo educativo en los consejos de la Federación de Estudiantes Universitarios: Con el fin de conocer mensualmente cuáles son las actividades que se han planificado, ayudarlos a que estas se materialicen y verificar el nivel educativo de las mismas y orientarlos con respecto a estas, además de hacerles llegar las inquietudes de los docentes.

11. Participación del grupo de trabajo educativo en las asambleas de brigadas: El trabajador educativo debe ser un participante activo en las mismas, siendo portavoz de la evaluación obtenida.

\section{Valoración de la pertinencia de la estrategia elaborada:}

Para valorar la pertinencia de la estrategia elaborada, se manejó el método de criterio de profesionales. Este método consiste en recoger información de las opiniones de un grupo de profesionales para obtener un consenso de opiniones y con ello criterios que permitan realizar una primera evaluación de la factibilidad de aplicación práctica de la estrategia elaborada, por ser directivos y profesionales implicados directamente en la actividad para la cual se elaboró la estrategia.

Para su aplicación se partió de la selección de un grupo de veinte profesionales que se consideraron capaces de ofrecer 
criterios sólidos acerca de la problemática de estudio, buscando en los mismos experiencia teórica y práctica con heterogeneidad de funciones y conocimientos.

Los profesionales seleccionados son decanos de las diferentes facultades, representantes de carreras, coordinadores de año y profesores especializados en dirección científica y trabajo educativo. Todos acumulan más de trece años en la profesión. De estos cuatro son doctores en ciencias, ocho máster y ocho concluyen maestrías.

A los profesionales seleccionados se les envió la propuesta de estrategia, donde se encuentra definida la estructura que la componen y acciones, además un cuestionario que los mismos contestaron de forma individual, en el cual se les pidió valorar la factibilidad de la estrategia en cuanto a estructura de la estrategia, contenido de la misión, visión, objetivo, acciones propuestas y criterios evaluativos. partieron:

Los criterios fundamentales emitidos

1. Sobre la estructura de la estrategia propuesta: El 100\% consideraron correcta la estructura de la estrategia, particularmente el hecho de contener la misión, la visión y objetivos, ya que no siempre se reconoce lo declarado en el contenido de estas.

2. Con respecto al contenido de la misión, la visión y objetivos, los profesionales consideraron novedoso lo declarado en estos elementos estructurales y son del criterio que se necesita profundizar en la preparación teórica y metodológica de los agentes educativos, los que incidirá en una mejor labor educativa y en el mejoramiento de todas las acciones que la enseñanza superior tiene hoy asignadas.

3. Concerniente a las acciones propuestas. El $90 \%$ de los profesionales consideraron suficientes las acciones propuestas y las valoran como de mucha utilidad para el mejoramiento de trabajo educativo en la Residencia Estudiantil y para el trabajo formativo en general con los estudiantes. El $10 \%$ de los consultados opinaron de manera favorable sobre las acciones, pero consideran que algunas de ellas pudieran ser rechazadas por los estudiantes y algunos profesores por no ser tradicional este trabajo en la Universidad de Holguín.

4. Relativo a las orientaciones de la tercera etapa de implementación, todos los consultados consideraron oportunas las recomendaciones emitidas para la etapa de implementación y seis de ellos consideran que se debe elaborar un folleto o manual donde se ofrezca la metodología para la realización de algunas de las acciones planteadas.

5. Referente a los criterios evaluativos, la totalidad de los consultados opinaron de manera favorable sobre los criterios de evaluación propuestos a pesar que consideran que algunos de estos criterios pudieran ser más argumentados. Todos los profesionales consideran pertinentes los criterios de evaluación pero consideran que se necesita profundizar más en ellos, incidiendo en un mejor control de la labor educativa.

6. Otros criterios recogidos de la valoración de los profesionales que se encontraron fueron:

- Consideran que la estrategia por su flexibilidad y adaptabilidad es posible aplicarla a las condiciones concretas de cualquier institución de la enseñanza superior, cumpliendo con las transformaciones que la misma tiene planteada.

- Además, que la propuesta es apreciada para la preparación de directivos de las diferentes estructuras universitarias y es considerada un gran intento para perfeccionar el proceso educativo en la institución. 
Asimismo, que permite la integración y retroalimentación del accionar de los órganos de dirección y técnicos de la Residencia Estudiantil para el cumplimiento de los objetivos previstos de forma sistémica.

\section{Conclusiones}

La culminación de estudio del proceso educativo en la Residencia Estudiantil permitió comprobar que el eslabón fundamental de la cadena de problemas que más ha influido en el trabajo educativo es la insuficiente cohesión de los agentes educativos que intervienen en la formación integral del profesional. Para responder al cumplimiento de los objetivos de la educación superior, se necesita cambiar las formas tradicionales por nuevas concepciones teóricas y metodológicas de lo más avanzado en el campo de la educación; es por ello que se tiene la exigencia de buscar vías más creativas para su perfeccionamiento y materialización. Por lo tanto, es necesario continuar con la preparación de los agentes educativos que intervienen en la formación integral de los estudiantes en los aspectos teóricos y metodológicos que brindan las ciencias psicológicas y pedagógicas para su labor educativa.

Se constató que la Estrategia de Integración constituye una herramienta que contribuye a la formación del futuro profesional desde el contexto residencial universitario.

\section{Notas}

1 Rodrigo Fuenzalida May, psicólogo, Pontificia Universidad Católica de Chile, profesor del centro de liderazgo de la Universidad Adolfo Ibáñez. Consultor en desarrollo organizacional. Valores Organizacionales.

\section{Referencias bibliográficas}

Augier, A. (2000). Metodología para la Elaboración e Implementación de la Estrategia Escolar en la Secundaria Básica. Universidad Pedagógica José de la Luz y Caballero. Holguín, Cuba.

Baster, E. (2002). La Escuela y el Proceso de Formación. La Habana, Cuba: Editorial Pueblo y Educación.

Benítez, M. (2003).La Familia Cubana. La Habana, Cuba: Editorial Ciencias Sociales.

Blanchard, K. y OConnor, M. (1997). Dirección por Valores. (2a. ed.).España: Ediciones Gestión 2000, S.A.

Boldiriev, N.I. (1982). Metodología de la Organización del Trabajo Educativo. La Habana, Cuba: Editorial Pueblo y Educación.

Castellanos, D. (2001). Atención a la Diversidad y Educación del Talento. Centro de Estudios Educacionales. Instituto Superior Pedagógico E. J. Varona. La Habana, Cuba.

Castellanos, D. (2003). Aprender y Enseñar en la Escuela. La Habana: Editorial Pueblo y Educación.

Castellanos, D. (2005). El Maestro Frente a la Diversidad. Centro de Estudios Educacionales. Universidad Pedagógica E. J. Varona. La Habana, Cuba.

Collazo, B. (1992). La Orientación en la Actividad Pedagógica. La Habana, Cuba: Editorial Pueblo y Educación.

Corso, J.; Colectivo de Autores S.A. La Formación de Valores en las Nuevas Generaciones. /S.L/ S.E.

Díaz , C. (2002). La Dirección por Valores en Acción a través de un Estudio de Caso. La Habana, Cuba.

Fleitas, R. (2005). Sociología y Política Social de la Familia. La Habana, Cuba: Editorial Félix Varela.

Fuenzalinda, R. Valores Organizacionales. Boletín gratuito distribuido por 
la Licenciada Márquez psicóloga Organizacional.

García, G. (2002). Compendio de Pedagogía. La Habana: Editorial Pueblo y Educación.

García, A. (2005). Dimensiones e Indicadores para el Diagnóstico Individual. Universidad Pedagógica José de la Luz y Caballero. Holguín, Cuba.

García, A. (2005). Métodos Educativos y de Orientación. Universidad Pedagógica José de la Luz y Caballero. Holguín, Cuba

González, D. (2004). Compendio Psicología Educativa. La Habana, Cuba: Editorial Pueblo y Educación.

González, A. (1995). Nociones de Sociología - Psicología-Pedagogía. La Habana, Cuba: Editorial Pueblo y Educación.

Hourruitinier, P. (2006). La Nueva Universidad Cubana: Un Modelo de Formación. La Habana, Cuba: Editorial Félix Varela.
Martínez, M. (1990). La Creatividad en la Escuela. Palacio de las Convenciones. La Habana, Cuba.

Martínez, M. (1995). Evaluación de la Creatividad del Maestro ¿Posibilidad o Realidad? Universidad Pedagógica Enrique José Varona. La Habana, Cuba.

Schukina, G.I. (1982). Teoría y Metodología de la Educación Comunista en la Escuela. La Habana, Cuba: Editorial Pueblo y Educación.

Torgella, G. (1998). Aprender a Vivir y a Convivir. La Habana, Cuba: Editorial Pueblo y Educación.

Vela, J. (2008). La Nueva Universidad: Necesidad Histórica y Responsabilidad Social. La Habana, Cuba: Editorial Félix Varela.

Valle, A. (2001). La Dirección Educacional. Apuntes. (Material en disquete) .La Habana, Cuba. 